\title{
Mitteilungen der Gesellschaft für Kognitionswissenschaft e.V.
}

\author{
Redaktion: Prof. Dr. Klaus Opwis, Institut für Psychologie der Universität Basel, \\ Bernoullistrasse 16, CH-4056 Basel (e-mail: opwis@ubaclu.unibas.ch)
}

\section{Mitgliederaufnahmen}

Folgende Personen wurden als neue ordentliche Mitglieder aufgenommen:

Barbara Becker (Sankt Augustin), Andreas Birk (Brüssel), Kerstin Dautenhahn (Hertfordshire, UK), Ingo Duwe (Schwerin), Günther Görz (Erlangen), Christoph Herrmann (Leipzig), Bernhard Hommel (Leiden), Christel Kemke (Soest), Hans Wilhelm Müller-Gärtner (Düsseldorf), Bernd S. Müller (Sankt Augustin), Bernhard Nebel (Freiburg), Marcus Plach (Saarbrücken), Jan Restat (Bremen), Wolfgang Schnotz (Landau), Norbert Seel (Freiburg), Silke Steininger (München), Markus Stöcklin (Basel), Hans Uszkoreit (Saarbrücken).

Folgende Personen wurden als neue assoziierte Mitglieder aufgenommen:

Cornelius Hagen (Freiburg), Raoul Sebastian John (Bochum), Peter Kuehn- lein (Bielefeld), Angela Nachtigall (Hamburg), Katharina Scheiter (Saarbrücken), Ladina Tschander (Hamburg), Silke Urban (Leipzig), Carmen Zahn (Tübingen), Markus Guhe (Hamburg).

Folgende Personen wurden als neue studentische Mitglieder aufgenommen:

Frank Borchard (Frankfurt), Julia Krause (Münster).

Die aktuelle Zahl der Mitglieder der Gesellschaft liegt nun bei 204 (Stand: Juli 2000).

\section{Veranstaltungen}

KogWis 2001

Die KogWis 2001 wird vom 25. bis 28. September 2001 in Leipzig stattfinden. Organisation: Angela Friederici, MPI für Neuropsychologische Forschung.
9. Herbstschule Kognitionswissenschaft, Freiburg, 10. bis 15. September 2000

Die Herbstschule lädt Studierende, Wissenschaftler und Interessierte aus den Bereichen Psychologie, Informatik, Computerlinguistik, Linguistik, Kognitionswissenschaft und verwandter Fachrichtungen ein. Die Herbstschule bietet Kurse, Plenumsvorträge sowie Methodenpraktika aus dem Bereich der Kognitionswissenschaft an. Die 9. Herbstschule für Kognitionswissenschaft wird gemeinsam von den Graduiertenkollegs „Menschliche und Maschinelle Intelligenz“ und dem ,Virtuellen Graduiertenkolleg" ausgerichtet.

Organisation: Michael Brenner, Katja Lay, Susanne Thalemann, Hans Spada und Gerhard Strube.

Informationen zum Kursprogramm stehen auf der WWW-Seite der Herbstschule zur Verfügung:

www.fr.vgk.de/herbstschule_2000 